\title{
CARENA, a tool to capture and replay web navigation sessions
}

\author{
I. J. Niño, B. de la Ossa, J. A. Gil, J. Sahuquillo and A. Pont ${ }^{\S}$ \\ Department of Computer Engineering. Polytechnic University of Valencia \\ Camino de Vera, s/n. 46071 Valencia (Spain) \\ \{innigon1,berospe\}@doctor.upv.es, \{jagil,jsahuqui,apont\}@disca.upv.es
}

\begin{abstract}
Web user behavior has widely changed over the last years. To perform precise and up-to-date web user behavior characterization is important to carry out representative web performance studies. In this sense, it is valuable to capture detailed information about the user's experience, which permits to perform a fine grain characterization.

Two main types of tools are distinguishable: complex commercial software tools like workload generators and academic tools. The latter mainly concentrate on the development of windows applications which gather web events (e.g., browser events) or tools modifying a part of the web browser code.

In this paper, we present CARENA, a client-side browser-embedded tool to capture and replay user navigation sessions. Like some commercial software packages our tool captures information about the user session, which can be used later to replay or mimic the gathered user navigation. Nevertheless, unlike these software packages, our tool emulates the original user think times since these times are important to obtain precise and reliable performance results. Among the main features of CARENA are: multiplatform, open source, lightweight, standards based, easily installable and usable, programmed in JavaScript and XUL.
\end{abstract}

\section{Introduction}

The main goal of Web performance research is to reduce the users' perceived latency. An important set of research has concentrated on techniques like caching or prefetching web contents. Many of them have developed simulation models to evaluate their proposals, using real or synthetic traces to carry out the experiments.

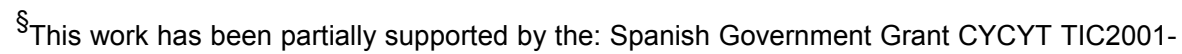
1374-C03-02 
Nowadays, due to the fast growth of the number of web users, studies focusing on the web server capacity planning or stressing are becoming more and more interesting for web server administrators. Capacity planning studies predict the server performance for a hypothetical increase of its workload volume. Stressing studies can be seen as the natural extension of capacity planning studies; these works predict the maximum number of users that a server can support at the same time without degrading its performance. These studies require a solid understanding of the workload and the users' behavior.

The most straightforward and accurate way to model a single user consists in instrumenting its own browser to collect the logs and other navigation characteristics. Furthermore, if the adequate information is collected, the gathered navigation session can be entirely and accurately replayed again. This fact enables that one gathered session could be replayed as many times as desired like some workload generators do [6][13][14].

The user behavior characterization can also be done by analyzing the log files obtained either in a proxy or in a web server. These files collect information about the web users' activity. Nevertheless, they usually collect data for a large set of users, so clustering techniques must be applied to identify typical patterns.

We analyzed a representative set of developed tools to capture web client behavior information (both commercial and academic), and we found some shortcomings that make them not suitable for our purposes. As a consequence we have developed an open-source tool, called CARENA (CApture and REplay NAvigations). It is a browser extension that captures the HTTP headers and visualizes them in real time. Our tool gathers precise information about the navigational user's behavior and has been implemented on the top of Live HTTP Headers Mozilla extension [1]. The CARENA code has been added in a modular way, which eases its migration to other Live HTTP Headers releases. The main features of CARENA are: i) it captures the user logs and additional information related with the navigation session, ii) it saves the navigation session into a XML structured file, iii) it permits to import navigation sessions and, iv) currently, it permits to replay in a precise way a single navigation session since it captures accurately the user think times. The captured sessions could be used to feed specific tools such as workload generators. See [13][14] for further details.

The remainder of this paper is organized as follows. Section 2 discusses the main $\log$ information characteristics. In Section 3 we discuss some related work. Section 4 explains the details of the proposed tool. Section 5, shows a CARENA working example. Finally, concluding remarks and future work are presented in Section 6 .

The remainder of this paper is organized as follows. Section 2 discusses the main $\log$ information characteristics. In Section 3 we discuss some related work. Section 4 explains the details of the proposed tool. Section 5, shows a CARENA working example. Finally, concluding remarks and future work are presented in Section 6. 


\section{Logging web user requests}

In order to obtain precise workload models different timing points of the web user sessions should be identified and captured; e.g., the starting of the document downloading. Log analysis can provide valuable information about the Web user behavior; although the collected information varies depending on the network point where the logs are collected; e.g., the server, the proxy or the browser.

Servers' logs usually collect information for a large set of users; therefore they collect much more information than logs gathered for a single web browser. To discern particular user behaviors from web servers' logs requires clustering techniques and specific software for analysis. In addition, the use of searcher engines makes difficult to distinguish between human navigations and robot navigations.

Like server's logs, proxy's logs also collect information for a large set of users; therefore, they also present the inconvenient that this fact involves. These logs collect information from both the client proxy side and the network side, and have been extensively used for web caching and prefetching studies.

Finally, since browser's logs collect navigation information for a single user, these logs are the most precise to characterize particular user' behavior. The main shortcoming is that monitoring browser navigations requires to instrument the browser code of the users that are part of the experiment, and to dispose the additional support for capturing, preparing and analyzing the extracted data by each browser. Despite all these advantages, few attempts have been made to instrument browsers because they do not usually offer open source code [12].

\section{Related work}

From the beginning of the WWW, researchers have concentrate on identifying the main features of user's navigations in order to characterize this workload, to detect user's behavior patterns, or for performance evaluation purposes. Despite this interest, only few tools have been developed to help researchers in these tasks.

The first attempts were proposed by Catledge and Pitkow in [9] and Tauscher and Greenberg in [10]. Both approaches instrumented the XMosaic Web browser. The main goal was to capture all user and browser events generated. Each activity record included timestamps, the visited URL, the page title, the final action, the invoking method and the user id, among other events.

In [9] Catledge and Pitkow studied the user behavior in order to understand their strategies when navigating the Web; for this purpose they analyze the log files from a client-side point of view. They calculated the time between each event for all events across the users, and determined session boundaries by analyzing these times, adopting the heuristic that a lapse of 25.5 minutes or greater indicated the end of a "session." Their study concludes with the characterization of user navigation patterns as serendipitous browsing, general browsing and searcher.

In [10] Tauscher and Greenberg studied the history mechanisms that Web browsers use to manage the recently requested pages. Their main interest was the 
analysis of re-visit patterns in the navigations. As a result they formulated some empirically-based principles of how users revisit pages using graphical browser features like the XMosaic.

The study developed by Choo et al [11], attempts to understand how the staff of seven different companies used the Web to seek information related to their daily work. To gather relevant information about WWW user navigations, they used a triangulation information approach, collecting information from three sources (e.g. questionnaire survey, web usage logs and personal interviews). To obtain the user's logs they developed the tool called Web Tracker. It is a typical Window application that watches the browser and collects menu choices, button bar selections, keystroke actions, and mouse clicks. All these actions are associated with the open Web page (URL). The browser actions are recorded in an ASCII text file. Combining the information obtained from the recorded logs with those data extracted from the questionnaires and interviews they were able to reconstruct the whole navigation process.

Reeder et al [12], developed a new tool called WebLogger which captures and records a significant number of user and application events during the browsing session. These captures are documented into a log file at three conceptual levels, the Input level (e.g. user actions on the mouse or keyboard), Interface level (e.g. user action on the interface elements of IE) and Application level (e.g. high-level actions of IE, such as retrieving an URL). This tools works under Windows operating systems and was developed to interact with the Microsoft Internet Explorer (IE) Web browser. The information recorded contains the event name, a list of specific parameters related with the event, the cumulative time elapsed since WebLogger was started (millisecond precision), the differential time elapsed since the previous recorded event (millisecond precision), and the current Windows system.

The HTTPLook [4] and ieHTTPHeaders [5] are shareware and freeware Internet tools respectively; developed to provide some information about the HTTP headers. These tools only work on Windows OS. The HTTPLook [4] sniffes the information transmitted between the Client and the Server. It captures and records information related with the web object type, the used technology, the IP address, messages arrival time and transmission time. ieHTTPHeaders [5] is an explorer bar developed for the Internet Explorer (IE) web browser that allows to show the HTTP Headers sent and received. Since to these two tools were not developed for research purposes they do not include analysis or statistics facilities.

Finally, a small set of web workload generators like [6] and [16] capture and record the user's logs and in order to obtain accurate information to partially generate the workload. Both are proprietary tools.

After this thorough study we can summarize that in general, the output file format of the tools mentioned in [4][5][9][10][12] is not so easy readable. Our tool CARENA solves this drawback because its output XML format helps the identification of all the elements that compose the navigation (e.g. frames, hidden 
frames, hole document, objects and attributes), the log file can be transformed into other generic format, (text plain, $\mathrm{rtf}, \ldots$ ) or a specific one. This last feature has special interest in the GUERNICA workload generator [13] [14].

The information captured is quite limited in most of the studied tools [4][5][9][11][12]. In general, just some users and browser specifics events are captured (e.g. mouse click, keystroke action, open file action). In contrast CARENA captures more relevant and accurate information about the network and the user; for instance: request and response HTTP headers size and time stamp, object size, user think time, status of whole document, document latency time stamp, start document load time stamp, end load document load time stamp, etc.

None of the discussed tools establish an estimation of the user think time. In some web workload generators this time is usually taken as a constant [13] while others estimate it by statistical distributions [14]. Unlike these tools, CARENA carefully and accurately calculates this time.

In this scenario our proposal provides important improvements at the same time it is presented as a open code tool. In the next section we present in detail the main architecture and features of CARENA.

\section{The CARENA solution}

CARENA is a tool to capture web navigation sessions at the client browser. This tool is implemented as a web browser extension that can easily be installed on any Mozilla or Mozilla Firefox browser independently of the underlying operating system. Through this tool the user is able to capture all the navigation session, including the page requests due to user clicks and their embedded objects. The navigation session is arranged around a XML structure and it can be later saved into a file, imported and replayed. Our work was done on top of Daniel Savard's Live HTTP Headers Mozilla extension [1]. Live HTTP Header is notified when Mozilla sends or receives HTTP requests or responses and captures the headers. It shows these HTTP headers on a window in real time, allowing to replay a single request, to edit it before replaying or to save the headers to a text file. In CARENA we added several new features to fulfill our requirements.

The main CARENA feature is that it retrieves additional information for each requested object. So, for each object we do not only retrieve its HTTP request and response headers, but also we timestamp when the request was sent and the response was received; we parse the HTTP headers to obtain the HTTP method and version, the response object size, the HTTP status messages, the object and referrer URLs and finally we also estimate the object latency from the request and response timestamps. If the user interrupts the navigation by clicking on the Stop button, a status attribute indicative of this fact is added to the representative XML structure of the webpage and all of the objects requested and not yet received.

The CARENA tool detects if an object was directly requested by the user or if it was an embedded object. Objects are grouped in document structures; each structure contains both the HTML object which URL that the user requested and 
all the objects retrieved due to the request. The information stored for every document includes among others the document URL, the number of retrieved objects, the time when the first object was requested and the time when the last object of the mass documents was received, the document latency, the document status indicating if any fail occurred, and the user think time. All this information can be saved to an XML structured file and imported at a later time. Those files can be easily treated or parsed by external tools since they are a plain text files with XML structure.

Navigation sessions can be later replayed emulating the original user behavior by requesting only the objects that the user really requested in its navigation session. Proceeding this way, Mozilla automatically retrieves all the embedded objects. The replay process takes into account the previously calculated user think time in order to defer the emulated user requests as in the capture session.

\subsection{Programming Environment}

Mozilla can be used by developers as a platform for creating applications that can be installed locally or run remotely over the Internet. It is a powerful and easy platform development framework to develop cross-platform applications. One does not need to get involved with the Mozilla source code to create a Mozilla application. A simple Mozilla binary that you download and install is the only development platform you need.

Currently does not exist an Integrated Development Environment (IDE), but it is possible to use many tools that make easy the application development. Some of these tools are: Venkman, DOM Inspector, XUL Maker, etc.

Venkman is a JavaScript debugger with support for breakpoints, local variable inspection, watch variables, single step, stop on error, code reformatting, etc.; and DOM Inspector is a tool for inspecting and editing the structure and widgets of the interface while the application is running, this was very helpful in the design and analysis of XML structures.

The Mozilla development framework is built around several technologies, thanks to the combination of these technologies, Mozilla allows developers to create applications on top of it. Mozilla structure is composed by two layers as Figure 1 depicts:

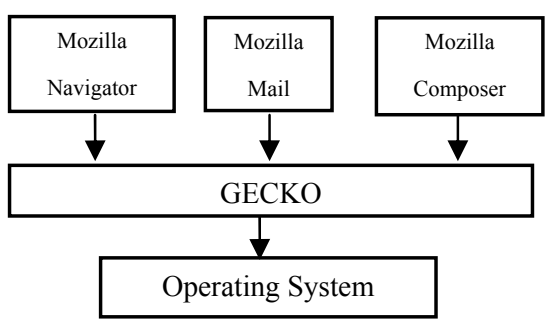

Figure 1: Mozilla Structure

Gecko is the software component in Netscape, Mozilla and Mozilla-based browsers that handles the parsing of the HTML, the layout of the pages, the document object model, and even the rendering of the entire application interface, 
is a fast, standards-compliant rendering engine that implements the $\mathrm{W} 3 \mathrm{C}$ DOM standards. Mozilla Navigator, Mozilla Mail, etc., are written in languages such as XUL, JavaScript, and XPCOM; therefore, Gecko is the interpreter that executes them. Some advantages of this are: modularity, platform independency and that the components can be added or removed easily. XPCOM and XPConnect are complementary technologies that enable the integration of external libraries with XUL applications.

XPCOM, which stands for Cross Platform Component Object Model, is a framework for writing cross-platform, modular software, which means that is a framework which allows developers to break up monolithic software projects into smaller pieces. These pieces, known as components, are then assembled back together at runtime. XPCOM components can be written in $\mathrm{C}, \mathrm{C}++$, and JavaScript, and they can be used from $\mathrm{C}, \mathrm{C}++$, and JavaScript. As an application, XPCOM uses a set of core XPCOM libraries to selectively load and manipulate XPCOM components [7].

XPConnect is a technology which enables simple interoperation between XPCOM and JavaScript. XPConnect allows JavaScript objects to transparently access and manipulate XPCOM objects. It also enables JavaScript objects to present XPCOM compliant interfaces to be called by XPCOM objects. In other words XPConnect is the bridge between JavaScript and XPCOM components as shown in Figure 2.

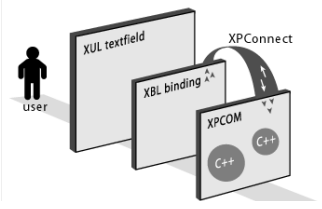

Figure 2: XPCOM/XPConnect

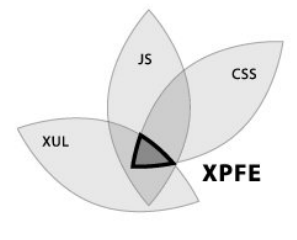

Figure 3: Cross-Platform Front End

XPFE (cross-platform front end) [2] was designed as a flexible interface working on any operating system. XPFE uses a number of existing web standards, such as Cascading Style Sheets, JavaScript, and XML (the XML component is a new language called XUL, the XML-based User-interface Language). In its most simple form, XPFE can be seen as the union of each technology. As shown in Figure 3. JavaScript creates the functionality for a Mozilla-based application, Cascading Style Sheets format the look and feel, and XUL creates the application's structure. Thanks to that XPFE is independent of the platform the applications created with XPFE will also be it.

XPInstall or Mozilla's Cross Platform Install provides a standard way of packaging XUL application components with an install script that Mozilla can download and execute. XPInstall enables users to effortlessly install new XUL applications over the Internet or from corporate intranet servers.

XUL (XML-based User-interface Language) Mozilla's XML-based User 
interface Language that lets build feature-rich cross platform applications that can run connected or disconnected from the Internet. It creates the structure and content of an application. The XUL language defines attributes that allow the programmer to define the actions controls react to. To define the dynamic behavior of the application, one can define JavaScript functions that will be called when certain user interface events happen.

JavaScript it is the core scripting language used in Mozilla. Three distinct levels of JavaScript are identified [2] as shown in Figure 4. The user interface level manipulates content through the DOM. The client layer calls on the services provided by XPCOM. JavaScript calls methods and gets data from scriptable components. Finally, the application layer is available to create XPCOM components.

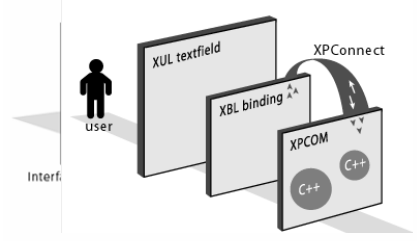

Figure 4: JavaScript Layers Architecture

The Mozilla DOM Core JavaScript interface implementation is an API to access HTML and XML documents. It provides web developers a structural representation of the documents and defines the way the structure is accessed by using JavaScript. Some methods like getElementById, getElementsByTagName, createElement, and createTextNode are provided by the DOM interface which permits querying and handling documents.

The Observer Service allows a client listener to register and unregistered for notifications of specific string referenced topics. Service also provides a way to notify registered listeners and a way to enumerate registered client listeners. The internal Mozilla notification system [3] helps to capture data. This system notifies the name of the event to the observer service, which deals with the lists of components watching for events. When the observer receives notification for an event, it passes that notification on to all listening components for that event. With this technique, the system simplifies the way to react when another component triggers an action.

CARENA deals with the following Mozilla events: object request, object response, document start, document end and document failure. Mozilla notifies CARENA when any of these events occurs; then, CARENA processes and stores the information on the internal DOM structure and shows the headers in the CARENA window.

\subsection{Capturing}

CARENA uses an XML object structure for the captured information. This structure is accessed trough the Mozilla DOM Core JavaScript interface 
implementation. HTTP request and response headers for a requested web object are stored into a JavaScript object that was created at the moment of the request. Headers are parsed to extract information like file size, referrer web object, etc., and stored as object's attributes. Timestamps are retrieved using the core JavaScript Date object method getTime that gets the system current local time.

When a web object is directly requested by the user, a new <document $>$ element is created into the XML structure, and a $<$ object $>$ element is added as the first child. New <object $>$ element children are created as soon as new requests are issued as a result of the current user request. When an HTTP response is received, CARENA locates the object in the XML structure where the HTTP request was previously stored and then new data is added. This means that each time the user clicks on a hyperlink, on a bookmark or types a new address into the address bar; a new < document> element is created in the XML structure; and all the embedded objects of that web document will be added as children.

When Mozilla reports that the document loading process has concluded (either successfully or as a result of an error occurred in the reception of the document) new attributes are added to the document element. Those attributes include the number of retrieved objects, the time when the last object of the document was completely received, the document latency, the document status indicating if some fail occurs or not, and the think time.

Frames and iframes, are stored as document children, like web objects but using a different XML tag. Since a document loaded on a frame or iframe can contain embedded web objects, frame elements can be parent of other objects.

When a HTTP response is a redirection (3XX status responses) we store the new URL for that object as an element attribute. If the browser decides to follow the redirection, the new request for the web object will be stored as a new object of the current $<$ document $>$.

\subsection{Saving and Importing}

Since we have the objects requests, responses and additional information stored in a XML structure, we can save it into a file. That file contains all the information available in memory, including headers, URLs, referrers, timestamps, latency, and the most important, the relation between objects, documents and frames.

The XML session file can be used by external tools to easily read, modify or even write their own navigation session files using existing XSL manipulation libraries. The XML file can be easily opened, read and parsed to retrieve all suitable information.

In order to import a saved session the user must select the XML file to import in a dialog window. After cleaning the XML structure, the XML file is loaded and parsed in order to fill up a new XML structure. During parsing, a content model based on the XML DOM is built [2]. Additionally the HTTP requests and responses are displayed in the headers window. Once the importing process has concluded, all variables will have exactly the same values they had when the file was firstly saved. 
Session XML files can be easily edited and modified by other tools before importing. This allows to modify previous navigation sessions think times, headers, requests, etc., before replaying the session and comparing the results.

\subsection{Replying}

The original Live HTTP Headers allow to select a HTTP header request, to modify it and send it again. In CARENA we added the possibility to replay the entire navigation session, not only a single request. This new 'Replay All' feature repeats the page HTTP request headers previously stored in the XML structure. The same URLs that the user directly requested when the navigation session was captured are now sent. CARENA does not request the original embedded objects that were retrieved when capturing the session; this job is done by the web browser that will retrieve any embedded object as usual. If the original website has not changed since capturing, then the embedded objects will be the same; otherwise, they probably will be different ones, depending on the changes that the website had had.

The original user think time is used to accurately replay the navigation session. This think time is usually a weakness in most current web workload generators; hence, it is usually simplified or taken as a constant. The user think time estimation is one of the main features of our tool, since it is precisely estimated in order to provide an accurate navigation replaying. As Figure 5 shows, the original user think time is calculated as the difference between the captured values of $t_{B}$ and $t_{A}$. Where $t_{\mathrm{A}}$ stamps the time in which the header part of the response arrives and the web page begins to render on the user screen, and $t_{B}$ represents the time at which the user submits a request for a new page. We could have calculated the think time as the difference between the time when the last object of a document is completely received and the next click, but due to the nature of incremental rendering of current browsers we selected the method explained before.

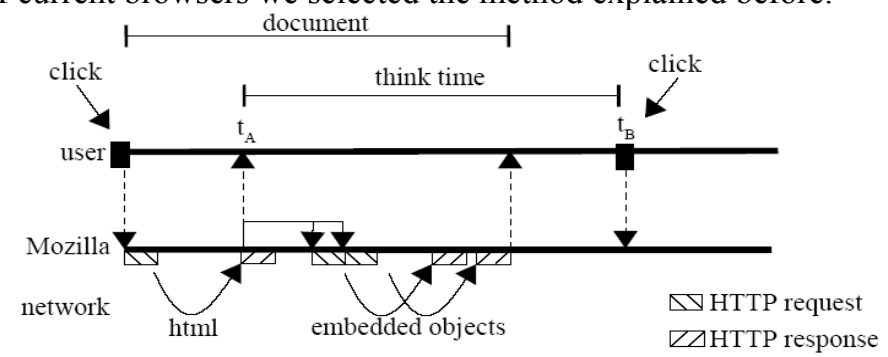

Figure 5: Details of user think time

The think time calculation may deviate in some situations. Some factors that may affect the precision are the file size, the number of embedded objects, the relevance of embedded objects, the user previous knowledge of the content, and the position of the relevant links in the webpage.

When replaying navigations, many website or network failures can rise; for instance, pages requested in original sessions may not exist in future sessions. In order to prevent these kinds of failures we use a timeout. When the timeout expires 
the tool assumes that the page is unavailable, therefore it executes the next request.

It is also possible to clear the browser disk and memory caches before starting the replay, in order to make it even more accurate.

\section{Working example}

Using CARENA to capture navigation sessions is extremely easy. It is started through the "Tools - Web developer" Mozilla menu. Once the Mozilla web browser and CARENA are open, any object the web browser requests to the network will be captured by our tool.

Figure 6 shows an example while retrieving the E2EMON05 website, HTTP headers are shown on the tool main window while browsing as soon as objects needed to compose the page are being retrieved.

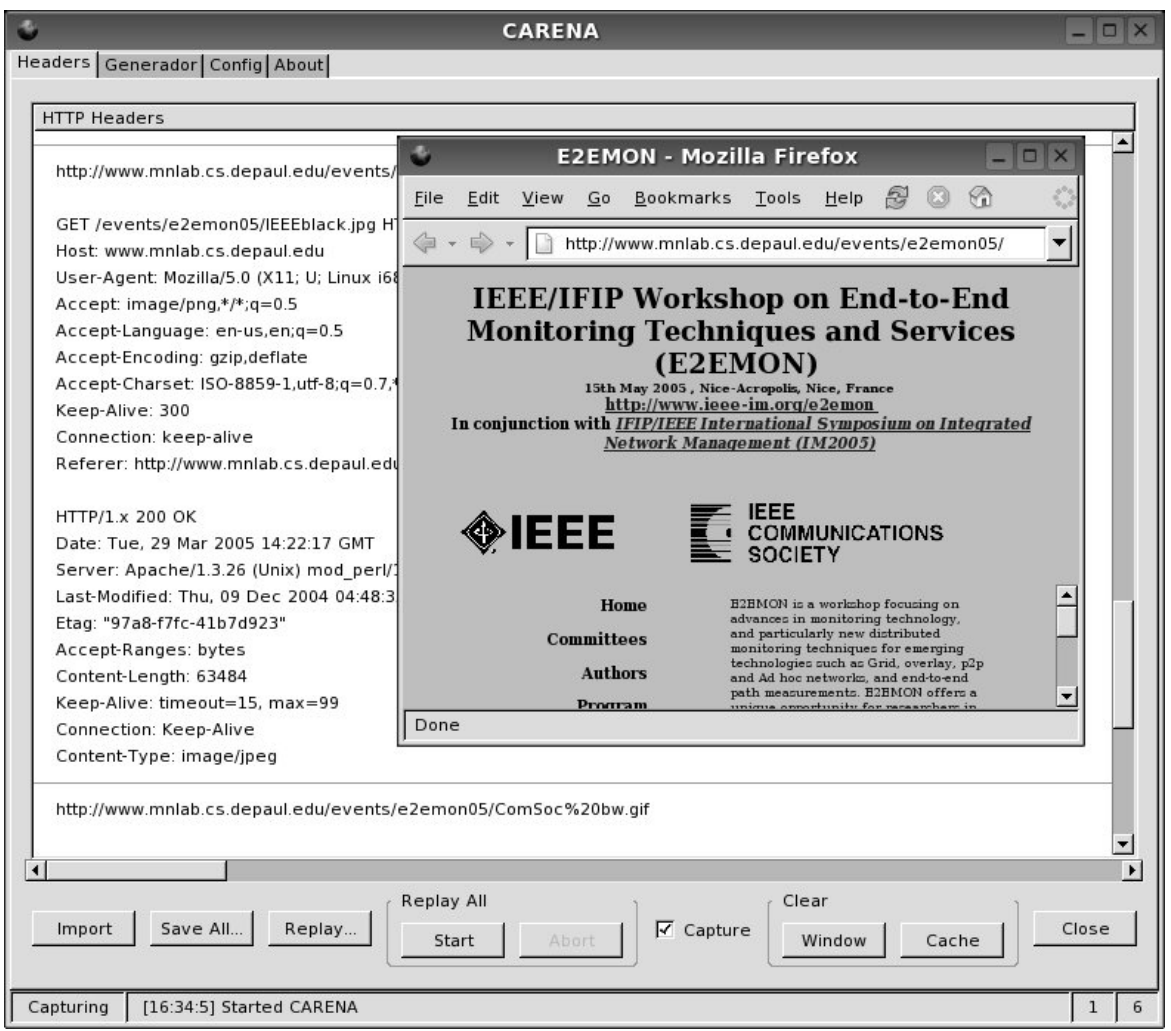

Figure 6: Navigating in Mozilla while CARENA captures the session

By using the "Save All..." button, the navigation session can be saved to an XML structured file that contains all the gathered information, including HTTP headers, timestamps and additional information. Figure 7 illustrates a navigation 
session where the user has requested three documents, as it is shown in the example, the first document requested is composed by three frames, and the first object of this document is the frame definition document while each document loaded in each frame appears into the $<$ frame $>$ label.

The "Import" button can be used to retrieve a saved session. And the replay of a single request can be achieved by selecting it into the main window and clicking into the "Replay..." button. The "Replay All" button allows replaying a whole session, the session replied can be the one being captured or a previously saved. Table 1 summarizes five repetitions of a session retrieving three documents, showing the deviations of the latency and think time from the original session; it reveals that while latencies suffer great deviations due to the instantaneous network and server load, the think time is more accurate, because it only depends on the load of the user machine.

Table 1: Deviations from the original session

\begin{tabular}{|c|l|c|c|c|}
\cline { 3 - 5 } \multicolumn{2}{c|}{} & \multicolumn{3}{c|}{ URI } \\
\cline { 3 - 5 } \multicolumn{2}{c|}{} & ./index.htm &.$/$ /organizers.htm &.$/$.importantdates.htm \\
\hline \multirow{4}{*}{$\begin{array}{c}\text { docLatency } \\
\text { deviation }\end{array}$} & repetition 1 & $0,00 \%$ & $-30,13 \%$ & $-30,13 \%$ \\
\cline { 2 - 5 } & repetition 2 & $9,30 \%$ & $-40,38 \%$ & $-30,13 \%$ \\
\cline { 2 - 5 } & repetition 3 & $8,31 \%$ & $-30,77 \%$ & $-19,87 \%$ \\
\cline { 2 - 5 } & repetition 4 & $10,36 \%$ & $-40,38 \%$ & $-30,13 \%$ \\
\cline { 2 - 5 } & repetition 5 & $9,30 \%$ & $-40,38 \%$ & $-30,13 \%$ \\
\hline \multirow{4}{*}{$\begin{array}{c}\text { docThinkTime } \\
\text { deviation }\end{array}$} & repetition 1 & $-0,42 \%$ & $0,54 \%$ & \\
\cline { 2 - 5 } & repetition 2 & $-0,71 \%$ & $3,93 \%$ & \\
\cline { 2 - 5 } & repetition 3 & $-0,99 \%$ & $0,57 \%$ & \\
\cline { 2 - 5 } & repetition 4 & $-1,70 \%$ & $1,11 \%$ & \\
\cline { 2 - 5 } & repetition 5 & $-0,99 \%$ & $3,93 \%$ & \\
\hline
\end{tabular}




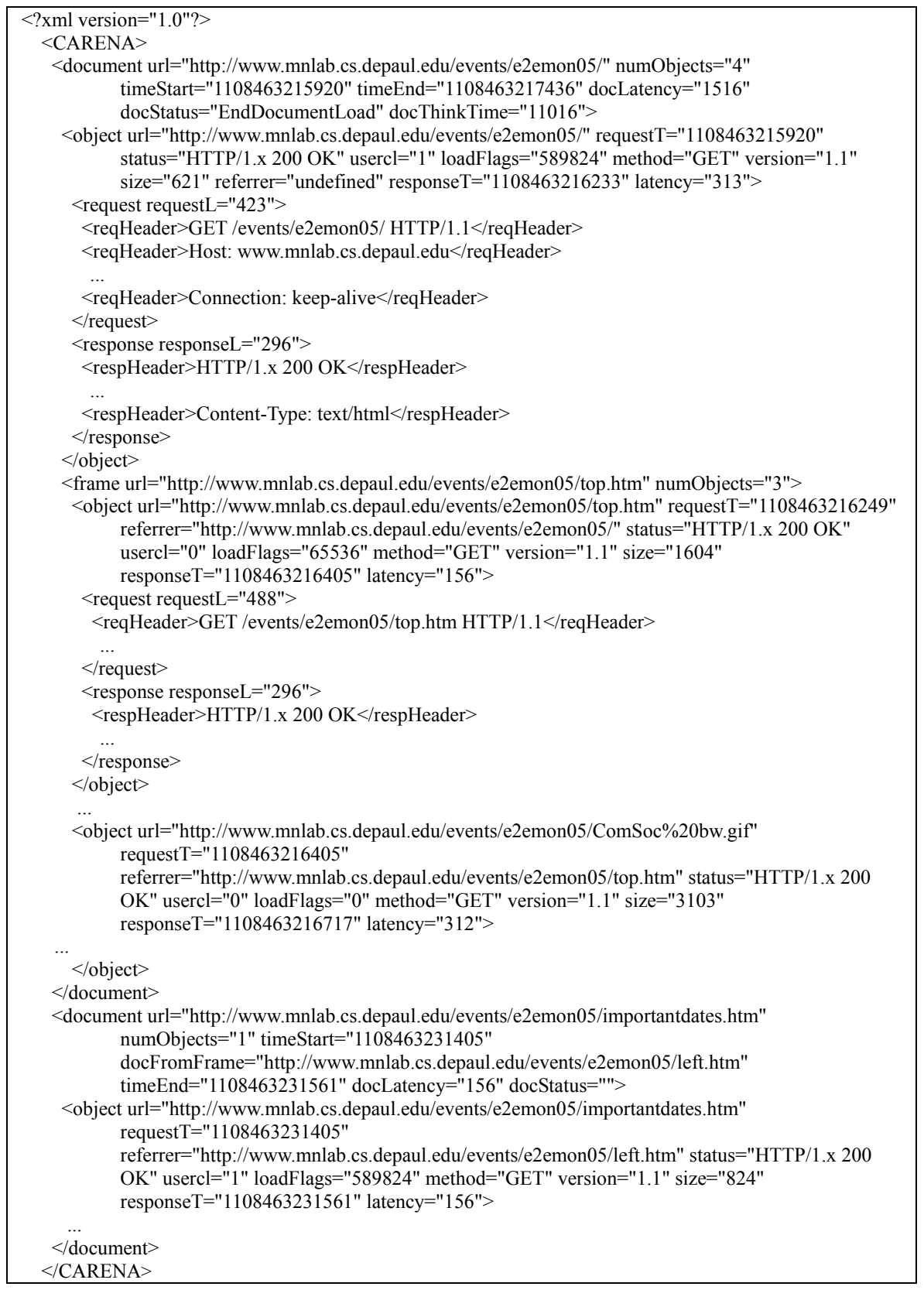

Figure 7: Part of the XML structured file 


\section{Conclusions}

In this paper we have proposed a tool to help web performance evaluation studies through a Mozilla extension that captures and replays browsing sessions. Our tool not only measures time related variables but also permits to accurately replay the complete navigation sessions.

The capture functionality can be used to get accurate data about the client workload, to characterize the user think time, or to know about the structure of the visited web pages as the number, type, size, etc. of embedded objects. Analyzing the captured navigation sessions a complete Web taxonomy can be performed. For instance: a) HTTP headers patterns and frequencies, b) Response times from the client point of view, considering the whole web page or each web object individually. c) web structure and content; for example, number of objects per page, object and page size, full page transmission time, and reutilization of objects in different parts of the Web site. d) web usage; analyzing how people access web pages and uses them in order to obtain the navigational behavior and also to discover the user access patterns, browser patterns, re-visit frequency of Web pages and user think time. With the above results, suggestions about design structure and usability of WWW pages, sites and browser can be done.

The replay functionality can be useful as a load generator for comparison purposes in a wide range of web performance research projects; for instance, proxy management algorithms or prefetching algorithms. It can also be used to automate functionality test processes of web applications.

The output file characterizes a real web navigation session, but other applications can be proposed. For instance: i) import and replay navigation sessions synthetically generated. ii) import previous navigation session and replay it in different network environments or conditions in order to compare the impact of those differences. iii) import a modified navigation session and replay it with the same network conditions in order to compare the impact of that modification.

The size of the navigation session file depends on the number of browsed pages, how many embedded objects do they contain and how many of those embedded objects are already cached on the browser. For instance, a page with 13 objects like http://www.ieee-im.org/ generates around 20 kilobytes, and a page with 40 objects like http://www.ieee.org/portal/ site generates around 60 kilobytes (an object approximately takes up 1500 bytes on XML formatted file).

With respect to the overhead introduced by CARENA, the CPU time is negligible with respect to the one used by Mozilla; the memory needed is barely noticeable since CARENA code contains less than 2.000 of JavaScript lines, and most of the requirements (i.e., XPCOM, XPFE, XUL, DOM, JavaScript machine) are already loaded and used by Mozilla for browsing purposes. Furthermore, memory consumption for data structures does not impose a problem when navigation sessions are shorter than several hours. 


\section{ACKNOWLEDGMENTS}

We would like to thank to Daniel Savard for the development of the Open Source Live HTTP Headers Mozilla extension in which we based our work, as well as all people that are involved in developing and improving Mozilla project.

\section{References}

[1] Live HTTP Headers website. http://livehttpheaders.mozdev.org/

[2] David Boswell, Brian King. Creating Applications with Mozilla. O'Reilly Editors. September 2002, http://books.mozdev.org

[3] Introduction to Mozilla source code. http://www.mozilla.org/hacking/codingintroduction.html\#xpcom

[4] Sniffer HTTPLook website. http://www.httpsniffer.com/

[5] ieHTTPHeaders website. http://www.blunck.info/iehttpheaders.html/

[6] RadView WebLOAD: http://www.radview.com. RadView Software. Recording WebLOAD ${ }^{\mathrm{TM}}$ Agendas. Version 5.0.

[7] Rick Parrish, An Introduction to XPCOM. Developer Works, SOA and Web Services. http://www-128.ibm.com/developerworks/webservices/library/coxpcom.html/

[8] Creating XPCOM Components. http://http://www.mozilla.org/projects/xpcom/book/cxc/html/quicktour2.html

[9] Lara D. Catledge and James E. Pitkow, , Characterizing Browsing Strategies in the World-Wide Web. Computer Networks and ISDN Systems, 27, 6 (April 1995), 1065-1073, 1995

[10] Linda Tauscher y Saul Greenberg, Patterns of Revisitation in World Wide Web Navigation, Proceedings of the SIGCHI conference on Human factors in computing systems, March 1997.

[11] Chun Choo, Brian Deltor y Don Turnball, Information Seeking on the Web: An Integrated Model of Browsing and Searching, Web Tracker: A Tool for Understanding Web Use, Asis Annual Meeting Contributed paper, 1999.

[12] Robert W. Reeder, Peter Pirolli, Stuart K. Card, Xerox PARC Tech. Report UIR-R-2000-06, 2000, WebLogger: A Data Collection Tool for Web-use Studies.

[13] Raúl Peña, Julio Sahuquillo, Ana Pont and José Antonio Gil, Modeling users' dynamic behavior in web application environments, 2nd International Working Conference on Performance Modeling and Evaluation of Heterogeneous Networks, 2004, 1-10 p.

[14] Raúl Peña, Julio Sahuquillo, Ana Pont and José Antonio Gil, Modeling users' dynamic behavior in e-business environments using Navigations International Journal of Electronic Business, 2005.

[15] Jakarta Apache. JMeter: http://jakarta.apache.org/jmeter/index.html

[16] Mercury LoadRunner. Mercury Interactive Corporation. http://www.mercury.com/us/products/performance-center/loadrunner/ 